\title{
An irregular lattice model to simulate crack paths in bonded granular assemblies.
}

\author{
C. M. Sands \\ University of Aberdeen, United Kingdom
}

\begin{abstract}
A 2D model of a bonded granular material is presented and its properties confirmed to be that of a brittle, isotropic elastic solid. The bond stiffnesses (axial tension/compression, shear and bending) are taken from the classical solutions to the external crack problem with two half-spaces bonded by a disc of intact material. An assembly of granules is simulated using a random array of points (representing the granule locations) with a prescribed minimum separation. The bonds are then generated by a Delaunay triangulation. This produces an isotropic array of bonds giving rise to a model material with isotropic properties. Crack growth is simulated by sequentially removing the most highly stressed bond in turn. Crack paths are then produced which are shown to agree with the predictions of linear elastic fracture mechanics, in respect of both the direction of propagation and the influence of specimen size. Some well-known problems are then simulated including: the interaction of two parallel cracks; diametrical compression of a disc; the four point bending of a beam; the influence of mortar strength on the behaviour of masonry; and a flat arch.
\end{abstract}

Keywords: granular, lattice, inter-granule bonds, lattice model,

\section{Introduction}

This paper focuses on a model developed to simulate the response of granular assemblies to mechanically induced stress. The granules are bonded only where they are in contact, and the bond strength is significantly less than the granule 5 strength. As such it can clearly be used to simulate the behaviour of many sedimentary rocks and a wide variety of ceramic materials, including some cement bonded blocks and many refractory products. The lattice model presented is, in effect, a sheet of three dimensional granules with all the bonds in the plane of the model. Consequently the model is currently only two dimensional; but the techniques used could easily be extended to three dimensions.

Lattice models have proved popular and have been used to simulate the behaviour of particulate materials generally [1, 2, 3, 4]; rocks [5, 6, 7, 8], and even a much wider range of brittle materials (e.g.concrete [9, 10, 11, 12, 13]) that 
do not have a microstructure that directly corresponds to the bonded granule idealisation.

Models of granular assemblies generally consist of:

- an assembly of granules with a lattice of bonds between (or near) the contacts;

- a prescription of bond stiffnesses;

- a prescription of bond strengths.

An assembly of granules and connecting bonds can be defined in a model in a number of different ways. Chandler 14 used a 2D hexagonal lattice of equally sized bonded spheres and created initial structural irregularities by randomly assigning some bonds to be missing. More recently, the positions, shape and ori25 entation of granules in the model material are commonly distributed randomly and arbitrarily, but a real material can be measured and then reproduced in the model [2, 5]. While many two dimensional models have been used successfully, three dimensional lattice models have also been developed [15, 16]. This approach has become more popular as computerised tomography has become more widely available.

Regular lattices in which all the elements have the same linear elastic properties have been shown to simulate linear elastic behaviour in the bulk material, however it has been shown [17, 9] that the crack pattern produced depends very significantly on the lattice layout. A random lattice produces a more realistic 35 result that appears to be independent of the particular lattice, but uniform material response commonly requires the properties of the elements to be adjusted to ensure that uniform strain results in uniform stress within the lattice. While that may present a problem for modelling perfectly homogeneous materials, it may not be an issue with materials that, while homogeneous at a large scale, 40 are not so at the scale of the lattice.

Many models simulate the bonds between the nodes of the lattice (regarded as the locations of the granules in the model presented here) as springs [18, 6, 7, 15], but beam theory is also used [1, 19, 20]. While most lattice models are based on linear elasticity, some have used viscous, cohesive crack, crack 45 bridging, tension softening and other non-linear approaches 21, 22, 23, 24 and some incorporate post-fracture compressive and frictional interaction 25, 26, 27]. While most lattice models use failure criteria based on stress, strain based systems have been used [19].

Lattice-like models using discrete element methods (DEM) have also been widely used 28, 8, 29, 30, 31 and although they have generally been limited to considering spherical or ellipsoidal granules, the bond stiffnesses are often similar to those used in more obviously lattice models. Discrete element methods also have the advantage that new contacts can be formed, and previously fractured bonds re-formed, that can pass shear and compression (though not tension) 55 between granules.

Several continuum models $32,33,34,35$ that can simulate crack growth in brittle materials have been developed based on a damage mechanics approach. 
Usually the stress and strain within the body is computed based on linear elastic theory and a threshold is set for the onset of damage. The elastic modulus is then permitted to degrade in accordance with a rule, or rules, that may be based on some mechanistic concept [32], be guided by empirical work 33], or by a widely accepted geomechanical model [34], or simply follow a realistic tension softening profile [35]. Although such models have advantages, such as the ease with which they can be incorporated into finite element analysis, they cannot

${ }_{65}$ capture the material behaviour at a fine scale. Lattice-particulate models, of the type presented in this paper have an advantage in that respect.

The model presented in this paper is based on the linear elastic theory for the bending, tension and shear of two half-spaces; and bond failure is assumed to occur in pure tension. It focusses on simulating crack path development by 70 sequentially breaking (and then removing from consideration) the most severely stressed bond. This is done one bond at a time, permitting stable crack growth to be simulated and also unstable crack growth in situations where inertial effects are negligible. The crack paths produced have been compared with recognised criteria [36] and observations [37, 38, 39]. Finally the model is used to explore 75 qualitatively the influence of mortar strength on the performance of structural masonry. In these simulations the model is used for the purposes of comparison only and the simulations do not relate to any specific material. It follows that any numerical values provided in respect of, for example, loads or displacements are not significant.

so A variety of different models have been used to simulate the response of masonry to applied load or foundation movement. Vertical loads were traditionally handled by considering the masonry as a no-tension structure and ensuring stability while avoiding local crushing of the material. More modern approaches take account of the bonding effect of the mortar joints. Pande, Liang and Mid-

${ }_{85}$ dleton [40], developed a procedure to establish orthotropic material properties for masonry panels which was then applied that to finite element analysis modified by a smeared crack approach [41]. Alpa and Monetto [42] applied theories relating to the frictional response of arrays of microcracks and considered walls with in-plane loading, while Anthoine [43] used the theory of periodic media to 90 establish homogenised properties. Luciano and Sacco [4] used a representative volume based on a masonry unit with adjoining mortar and considered the possible crack paths that could form and the consequent states of the masonry to determine characteristic moduli for each state. Homogenization techniques were then adopted to establish a nonlinear constitutive law for the masonry material.

A common approach for thin walls subjected to lateral loads treats masonry panels as plates [45, 46, 47], and that approach can be extended to include vertical loads [48]. Another technique [49, 50] uses discrete element methods, often supported by homogenization techniques and the use of representative volumes. While these approaches have several advantages, they lack the simplicity of the model presented here. 


\section{Construction of the lattice}

\subsection{The nodes and bonds}

In the model presented here, random points are generated in succession and discarded if a new point falls outside a defined area or is too close to any other existing point. In this way an irregular, but reasonably evenly distributed set of points is created. Each of these points represents the location of the centroid of a granule and forms a node in the lattice. Each node can translate in two dimensions and rotate. A Delaunay triangulation is then performed to define which nodes are connected to a neighbouring node. Each bond connects two nodes and the granule to granule bond is assumed to exist halfway between the two connected nodes. The model, therefore, models non-spherical granules.

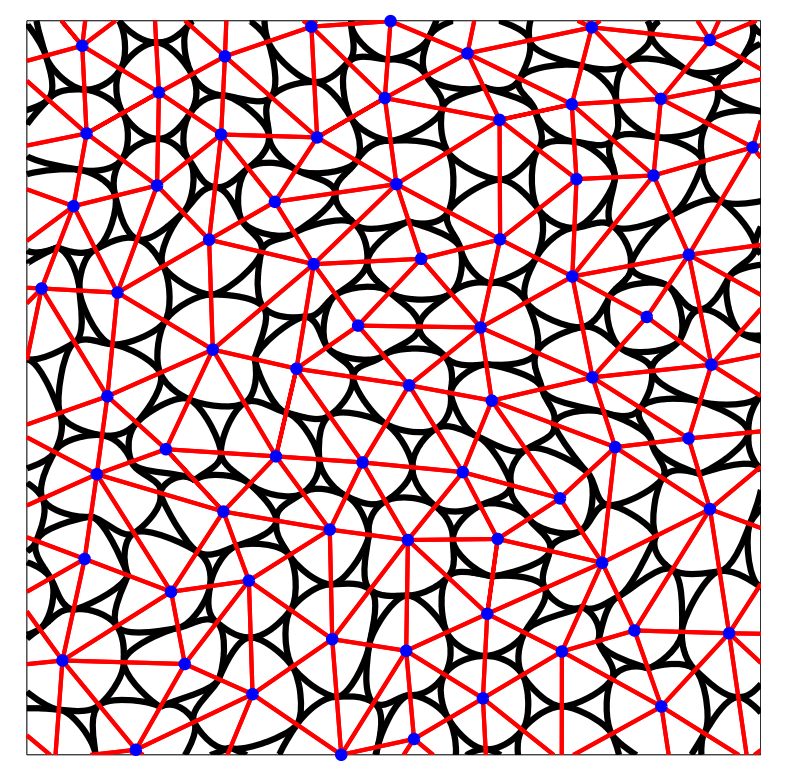

Figure 1: A typical granular assembly (shown as black lines) with the structure of the model representing the assembly superimposed. The nodes that represent the grains are shown as blue dots, and the lattice members that encapsulate the bonds are shown as red lines. The nodes act at the centroid of the grains and the contacts are taken as acting at the mid-point of the lattice members that connect the nodes.

Figure 1 illustrates a typical granular structure of the sort being simulated in the work presented here, overlain by the lattice structure used to simulate the granular assembly. The blue dots are nodes in the lattice and each node 115 represents the centroid of a grain. The bonds between the grains are represented by the lattice members (shown as red lines) and the contacts between the grains are assumed to act at the mid-point of the lattice member. In the simulations presented in this paper the area of the bond connecting two grains was, on 
average, about $1.5 \%$ of the surface area of each of the grains that the bond connected, with a maximum of $3.7 \%$; justifying the assumption that the contact area was small. The diameter of the grains was, on average, about $1.2 \%$ of the size of the body being simulated.

\subsection{The bond stiffness}

The area of each bond is assumed to be small in comparison to the surface area of the granules. The bond stiffnesses (axial tension/compression, shear and bending) are taken from the classical solutions to the external crack problem with two half-spaces bonded by a disc of intact material. Barber [51] gives expressions for the case of the related problem of a cylindrical rigid punch of radius $a$ that is pushed, by a normal compressive force $-t$, a distance $-e_{x} / 2$ 130 into an elastic half space that has shear modulus $G$ and Poisson's ratio $\nu$.

$$
t=\frac{4 G a\left(\frac{e_{x}}{2}\right)}{(1-\nu)} .
$$

Bending stiffness is found by considering the case of a punch that is tilted through an angle $\phi / 2$ by a moment $m$ such that

$$
m=\frac{8 G a^{3}\left(\frac{\phi}{2}\right)}{3(1-\nu)} .
$$

Shear stiffness is found (using Johnson [52]) by considering the tangential force $q$ required to produce a tangential displacement, $e_{y} / 2$, of a granule

$$
q=\frac{4 G a}{(2-\nu)}\left(\frac{e_{y}}{2}\right) .
$$

135 of the two half-spaces, in terms of Young's modulus $(E)$ and Poisson's ratio $(\nu)$ as follows

$$
\frac{E a}{\left(1-\nu^{2}\right)}
$$

for the axial case,

$$
\frac{2 E a}{((2-\nu)(1+\nu))}
$$

for shear, and

$$
\frac{2 E a^{3}}{(3(1-\nu)(1+\nu))},
$$

for rotation. 


\subsection{Developing the local stiffness matrix}

Consider two granules, sitting a distance $L$ apart, in a two dimensional Cartesian coordinate system, with the local $x$ axis passing through the nodes of both granules and the centre of the bond that is located half way between them.

145 The granules are subjected to axial, shear and bending forces that are applied to the nodes of the lattice. Following the procedure presented by Livesley [53], the local stiffness matrix for the lattice member was constructed from an array of primitives:

$$
\mathbf{P}=\left[\begin{array}{ccc}
\frac{E a}{\left(1-\nu^{2}\right)} & 0 & 0 \\
0 & \frac{2 E a}{(2-\nu)(1+\nu)} & 0 \\
0 & 0 & \frac{2 E a^{3}}{3(1-\nu)(1+\nu)}
\end{array}\right]
$$

based on the stiffnesses outlined in Section 2.2 Using the conventional approach of splitting the full stiffness matrix into four sub-matrices,

$$
\mathbf{K}=\left[\begin{array}{ll}
k_{11} & k_{12} \\
k_{21} & k_{22}
\end{array}\right] \text {, }
$$

and the full matrix, $\mathbf{K}$, can be obtained from the sub-matrices using

$$
k_{i j}=\mathbf{H}_{i} \mathbf{P} \mathbf{H}_{j}^{T},
$$

where $i$ and $j$ can take the values 1 or 2 , and

$$
\mathbf{H}_{1}=-\left[\begin{array}{ccc}
1 & 0 & 0 \\
0 & 1 & 0 \\
0 & L / 2 & 1
\end{array}\right]
$$

and

$$
\mathbf{H}_{2}=\left[\begin{array}{ccc}
1 & 0 & 0 \\
0 & 1 & 0 \\
0 & -L / 2 & 1
\end{array}\right] .
$$

The stiffness matrix thus developed can then be used following the classic approach: $\mathbf{f}=\mathbf{K u}$; where $\mathbf{f}$ is the vector of nodal forces; $\mathbf{K}$ is the stiffness matrix and $\mathbf{u}$ is the vector of nodal displacements.

\section{Testing the Model}

\subsection{Applying the Boundary Conditions}

160 The boundary conditions were applied by selecting those boundary nodes that would be given prescribed displacements. The analysis proceeded in the conventional manner for a standard stiffness method problem and the loads at each bond were evaluated. All the simulations presented in this paper used prescribed displacements and did not proceed by applying loads to any of the 
nodes. The forces required to produce those deformations were calculated (when required) from the vector of nodal forces produced by the simulation. As the granule to granule connectivity was defined in terms of a 'beam like' stiffness matrix, granule rotation could be prescribed by applying a rotation to the relevant end of the appropriate 'beam'. It should be recalled that the simulations are comparative only as no specific material is being simulated.

\subsection{Checking Isotropic Behaviour}

It was necessary to ensure that a random array of bonded granules would produce an assembly that manifested an isotropic response. Such arrays were generated as described in Section 2.1 and the orientation of the bonds was

175 checked by constructing polar histograms of the orientation of the elements that formed the Delaunay triangulations. The bonds to be examined were selected randomly using a Matlab algorithm and a large number of bonds were examined for several different granule arrays. Typical histograms are shown in Figure 2. These checks demonstrated that the procedure outlined in Section 2 would produce a truly isotropic array.

\subsection{Linear Elastic Behaviour}

The inter-granular bonds obey Hooke's law. The constitutive relationships between stress, $\sigma$, and strain, $\varepsilon$, for a two dimensional system are shown below.

$$
\begin{gathered}
\varepsilon_{x x}=\frac{\sigma_{x x}}{E}-\frac{\nu \sigma_{y y}}{E} \\
\varepsilon_{y y}=\frac{\sigma_{y y}}{E}-\frac{\nu \sigma_{x x}}{E} \\
\varepsilon_{x y}=\frac{\sigma_{x y}}{2 G},
\end{gathered}
$$

where the subscripts relate to the $x$ and $y$ axes in the usual manner.

The mechanical response of granular assemblies constructed as described in Section 2 has been reported [54] to be consistent with a 2D isotropic linear elastic solid.

\subsection{Simulating Fracture}

The work described in the previous section indicated that initially the bulk behaviour of the assembly was isotropic. The bond between two adjacent grains was assumed to occur at the mid-point of the lattice member joining the nodes representing each grain. It follows that the bond stresses were not uniform. The area of the contact between two grains was assumed to be small compared to the size of the grain. It can be seen from Section 2.2 that the size of the contact has much greater influence on the bending stiffness than on the shear or axial stiffness.

Fracture was simulated as follows. First the displacements at the support points were constrained to provide appropriate boundary conditions. An appropriate point, or set of points, $P$, on the body was given a set displacement, $U_{\text {set }}$, 


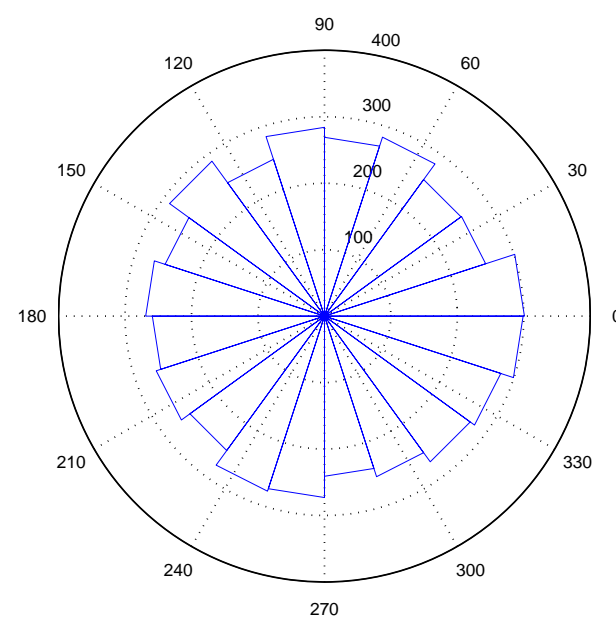

(a)

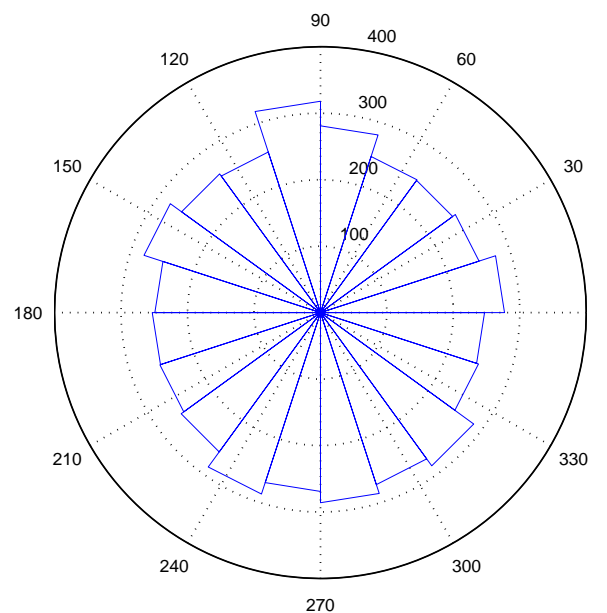

(c)

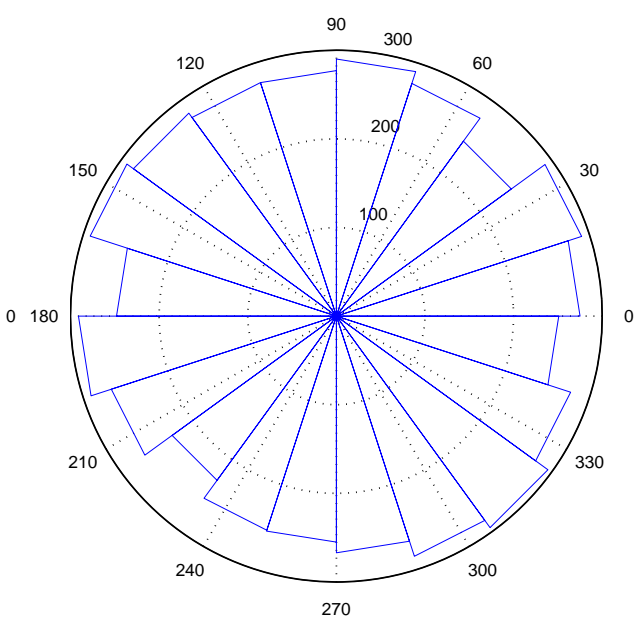

(b)

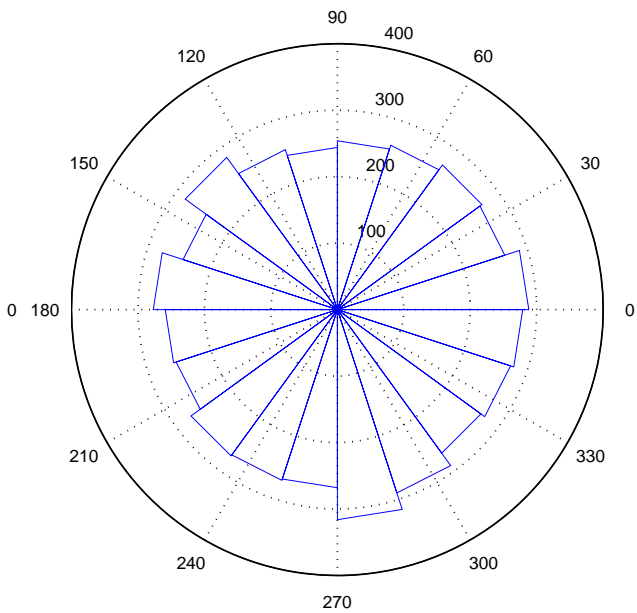

(d)

Figure 2: Polar Histograms of Bond Orientations. 
and the reactions $(F)$ determined. The bond tensions, $T$, were determined and used to calculate $\lambda=T / S$ (where $S$ is the bond strength) for each bond. The bond with the maximum value of $\lambda$ (denoted as $\lambda_{n}^{\max }$, where $n$ related to the 205 simulation step) was then recorded as being broken and its stiffness subtracted from the appropriate elements of the stiffness matrix. $F_{n}$ and $\lambda_{n}^{\max }$ were stored and the modified lattice re-analysed and the process repeated. Clearly the stiffness of the body being simulated reduced with each fracture. The simulation continued until the body was deemed to have completely failed.

The load versus displacement plots were then created. Prior to loading the displacement was zero, and the displacement associated with the first fracture, $\lambda_{1}^{\max } U_{\text {set }}$, was plotted against the value of $\lambda_{1}^{\max } F_{1}$, with the slope of the plot equivalent to the initial stiffness of the undamaged body. Fractures that occur in the simulation after that first fracture sometimes produced values of $\lambda_{n}^{\max } U_{\text {set }}$ that were smaller than $\lambda_{1}^{\max } U_{\text {set }}$. These were part of an unstable fracture process initiated by the first fracture. The next fracture for which the value of $\lambda_{n}^{\max }$ exceeded the previous maximum was then established and $\lambda_{n}^{\max } F_{n}$ versus $\lambda_{n}^{\max } U_{\text {set }}$ plotted, ignoring any unstable fractures. The 'step' changes in the stiffness of the body as fracture progressed is evident in the plots shown

220 in Figure 11, 13 and 14 and illustrates the tension softening known to occur in materials of the type being simulated in the work presented here.

\subsection{Propagation of Straight Crack}

A standard crack test was simulated by first constructing a rectangular assembly of randomly distributed granules. A small section of bonds located at the

225 mid-width and near the top of the assembly were removed from consideration in the loading algorithm, as illustrated in Figure 3. The elements removed have been shown as a thick black line for clarity. The boundary nodes were prevented from moving vertically, and the boundary nodes at either side were displaced horizontally outwards to encourage the pre-existing crack to grow. The crack 230 extension predicted by the model is as expected and is shown in Figure 4, on the un-deformed geometry.

An examination of the bond forces during the simulations revealed much higher forces in the bonds at the crack tip, consistent with the expected stress concentration at this position, and near uniform strain in the uncracked portion.

235 Results of dimensional analysis predict that, for the test described above carried out on a sample of width $W$, a relationship exists between the total force $(F)$ applied to each long edge, of intact length, $L$, such that $\sqrt{W}$ is proportional to $F / L$. A large number of tests were carried out [54] using the model presented here for samples of various widths $(W)$ and the results are illustrated in Figure 5

240 This confirms the linear relationship between $F / L$ and $\sqrt{W}$.

\subsection{Propagation of Kinked Crack}

The behaviour of the model when propagating a kinked crack was investigated by setting up a sample, pre-cracked for a set length, generally as indicated in Figure 3. For this test the nodes on the right hand vertical boundary of the 


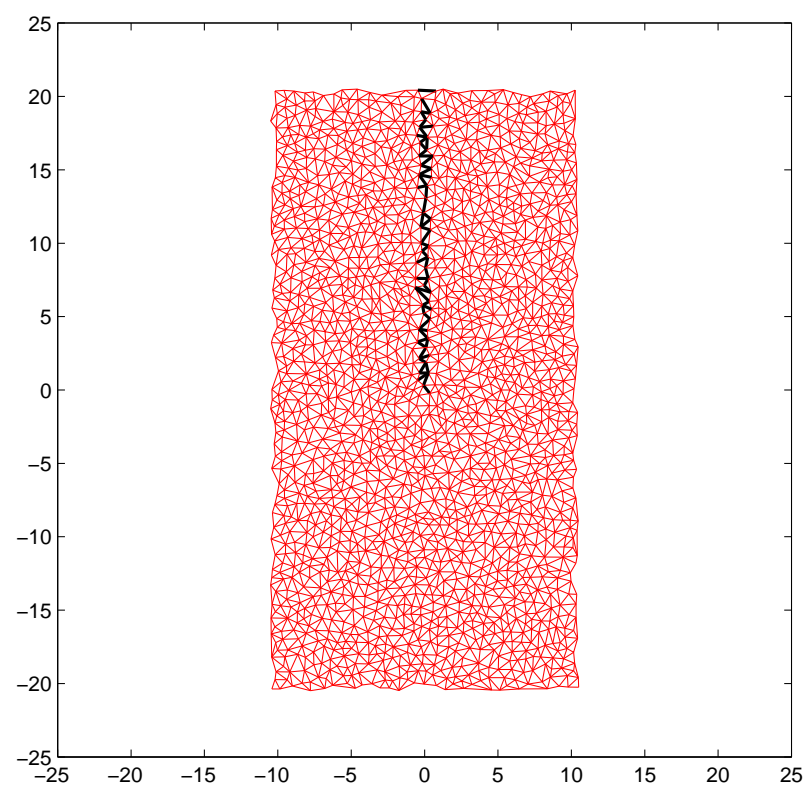

Figure 3: Rectangular test sample with pre-crack shown as thick black lines.

assembly were displaced outwards and upwards, while those on the left hand vertical boundary were displaced outwards and downwards, but were free to rotate. All the other nodes were free to displace and rotate in response to the displacement of the boundary nodes. The resulting crack propagation is shown in Figure 6. This test simulates Mode II cracking in the kinked portion of the crack (i.e. ignoring the section that was pre-cracked before the application of any load).

Classic fracture mechanics [55] provides the following relationship

$$
\sigma_{\theta}=\frac{1}{(2 r)^{\frac{1}{2}}} \cos \frac{\theta}{2}\left[K_{I} \cos ^{2} \frac{\theta}{2}-\frac{3}{2} K_{I I} \sin \theta\right],
$$

where $\sigma_{\theta}$ is the hoop stress at an angle $\theta$ from the pre-existing crack and a distance $r$ from the crack tip; and $K_{I}$ and $K_{I I}$ are the stress intensity factors for Mode I and Mode II loading respectively. A large number of simulations were then carried out for a particular granular arrangement containing a crack as shown in Figure 3. The assembly was then subjected to a variety of deformations consisting of a combination of horizontal and vertical displacement of the two vertical boundaries. This pattern of deformation produced kinked cracks of 260 the form shown in Figure 6. The angle that the kinked portion of the crack made with the vertical drawn through the initial crack was measured from the plotted figures showing crack growth. The values of $K_{I}$ and $K_{I I}$ were calculated from the following formulae, derived using the procedure presented by Rice in 


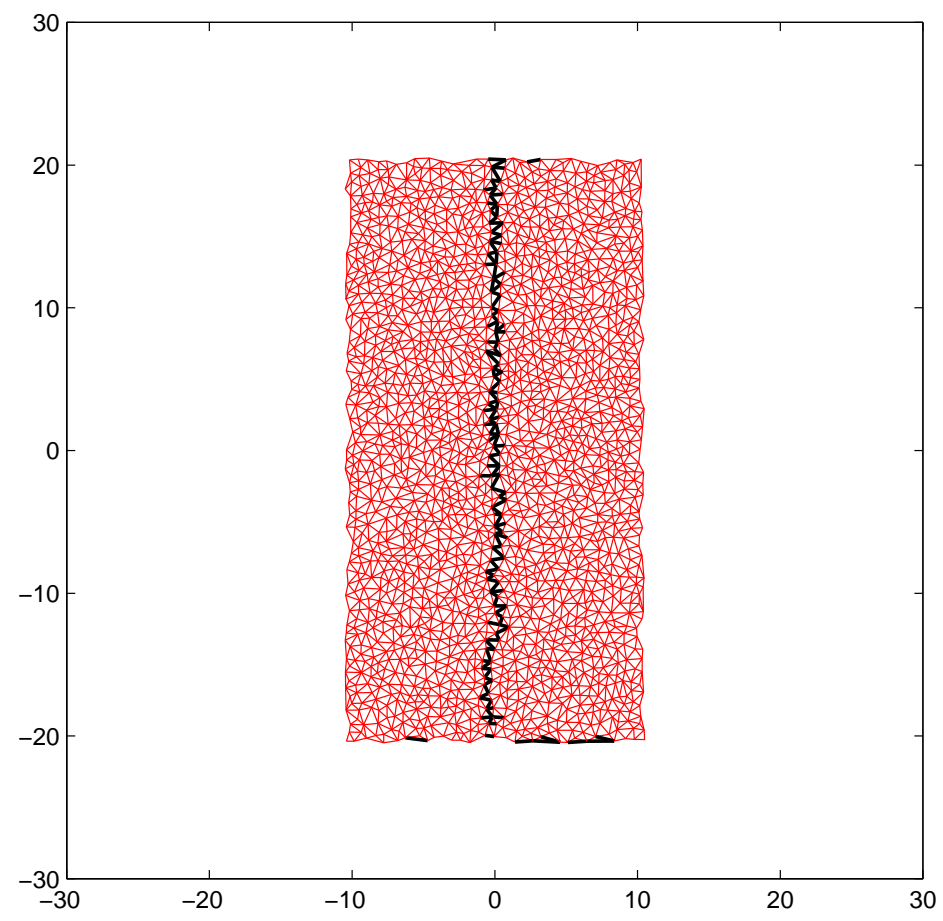

Figure 4: Rectangular test sample after crack extension. Cracks shown as thick black lines.

a discussion [56] on a paper by Knauss [57].

$$
K_{I}=\sigma_{11}(1-\nu)^{\frac{1}{2}} h_{w}^{\frac{1}{2}}
$$

and

$$
K_{I I}=(1+\nu)^{\frac{1}{2}} \sigma_{12}\left(2 h_{w}\right)^{\frac{1}{2}}
$$

where $\sigma_{11}$ is the stress at the crack tip that tends to open the crack in mode I, $\sigma_{12}$ is the shear stress at the crack tip, $\nu$ is Poisson's ratio (taken as 0.19 for these simulations, following work by Adaiem [54]) and $h_{w}$ is half the width of the sample. This permitted a parameter $\mu$ to be determined

$$
\mu=\frac{\left(\frac{K_{I}}{K_{I I}}\right)}{\sqrt{\left(\frac{K_{I}}{K_{I I}}\right)^{2}+1} .}
$$

The values of $K_{I}$ and $K_{I I}$ were determined as outlined above, from the boundary reactions, during the first loading run of the simulation (i.e. at the onset of crack propagation). This was repeated for each deformation arrangement (i.e. for a range of values of $\mu$ ) and for two different assemblies. The 275 results obtained from the simulations are compared with the theory in Figure 7 


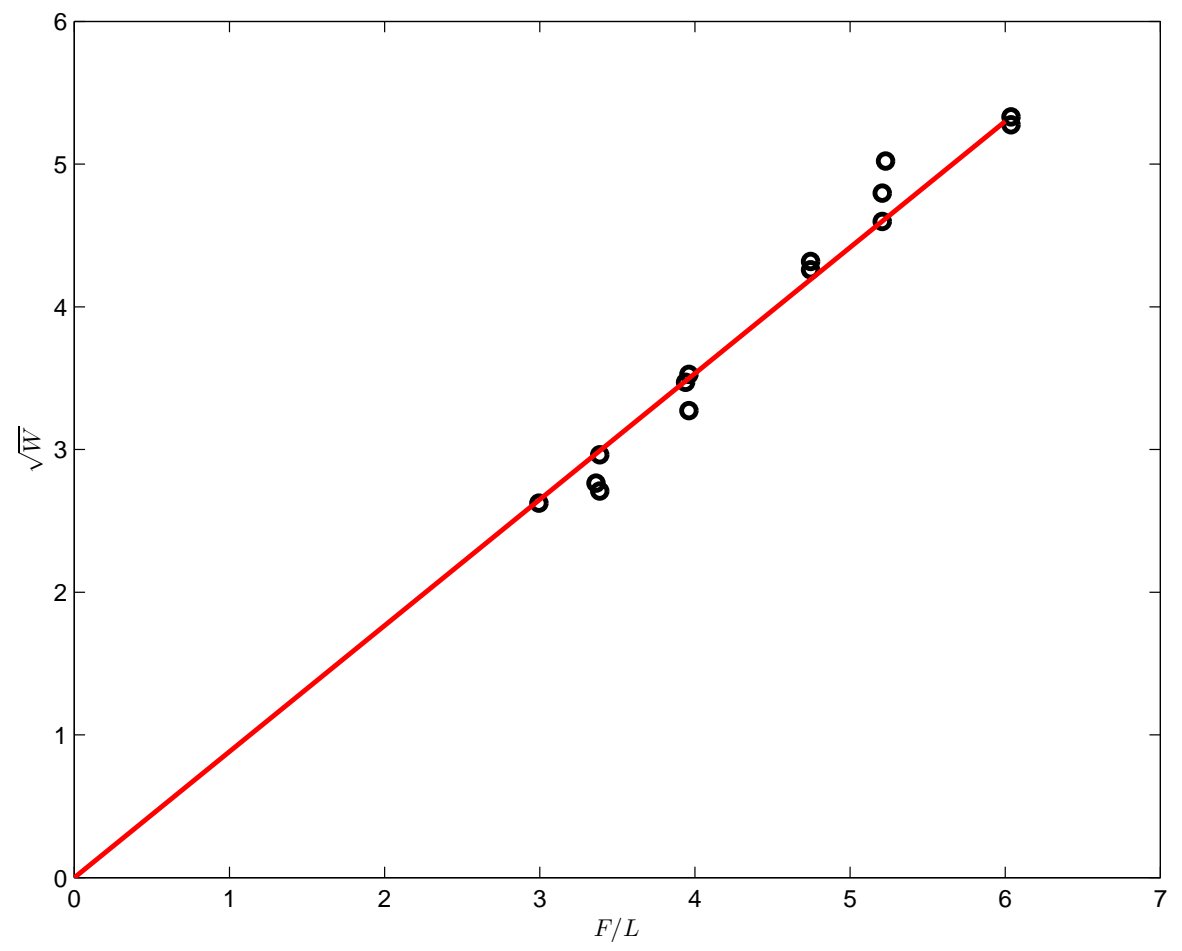

Figure 5: Confirmation of the linear relationship between $F / L$ and $\sqrt{W}$ where $F$ is the total force applied to each long edge, of intact length $L$.(Redrawn from [54].)

It is clear from Figure 7 that, as one would expect, the model behaviour is influenced by the granular arrangement and the significance of this was investigated by running simulations using different randomly created granular arrangements for the same deformation mechanism. Deformation mechanisms associated with $\frac{K_{I}}{K_{I I}}=0.0$ and $\frac{K_{I}}{K_{I I}}=1.0$ were explored and ten assemblies created for each mechanism. The first of these produced (for the first deformation mechanism) an average $\frac{K_{I}}{K_{I I}}=-0.0250$ and a variance of 0.0064 , while the second produced (for the second deformation mechanism) an average $\frac{K_{I}}{K_{I I}}=0.9499$ and a variance of 0.0024. A third set of randomly generated assemblies was created using a denser packing mechanism and evaluated using the second deformation mechanism. This resulted in an average $\frac{K_{I}}{K_{I I}}=0.9799$ and a variance of 0.0012 . The procedure was then repeated (again for the second deformation mechanism), in an attempt to place more granules within the sample boundaries in order to achieve a greater packing density. This produced an average

$290 \frac{K_{I}}{K_{I I}}=0.9421$ and a variance of 0.0022 . Clearly, the number of granules that can be placed within a set boundary depends on the limit placed on the minimum granule size and these results suggest that the initial simulations were close to 


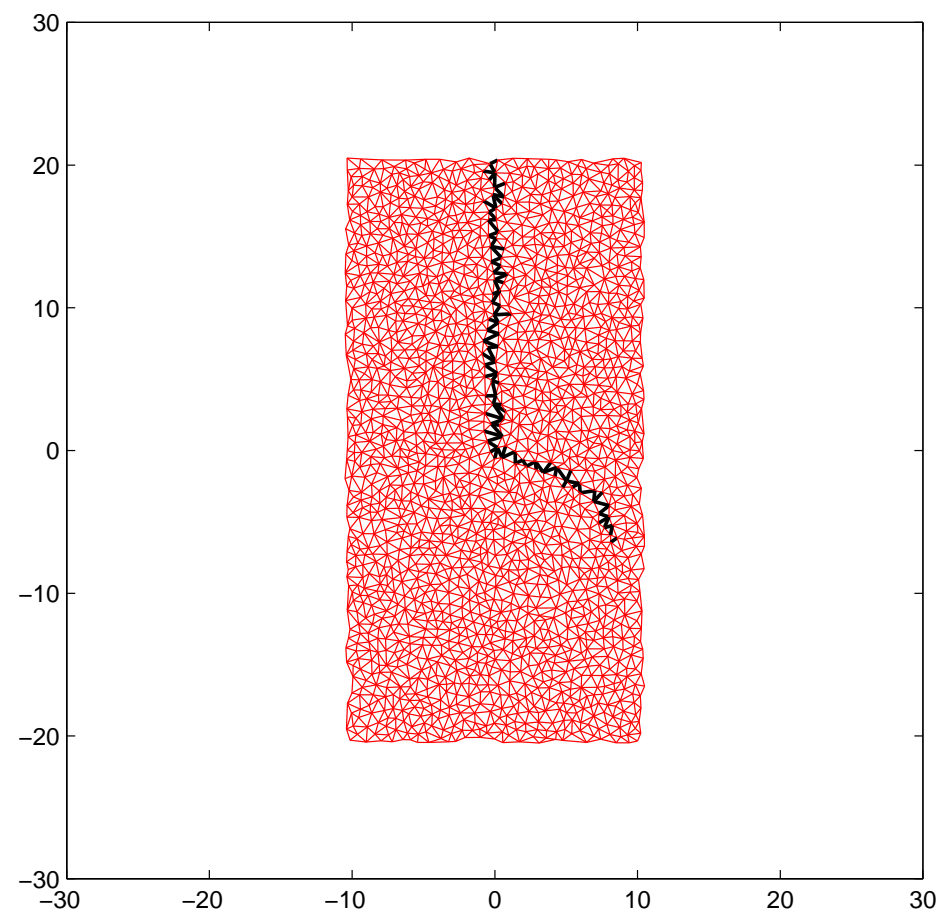

Figure 6: Propagation of kinked crack. Cracks shown as thick black lines.

the optimum for the granule size chosen.

These tests indicate that the model captures the essential features of linear elastic fracture mechanics and can simulate the behaviour of real, weakly bonded granular assemblies (such as sedimentary rocks, lean mix concrete and concrete formed using strong aggregate, and several types of ceramic formed from strong grains - such as grinding wheels).

\section{Predictions Using the Model}

\subsection{Propagation of two Opposing Off-set Cracks}

The model was then used to explore the response of two opposing cracks, off-set from each other, when the bond strength is uniform. Four arrangements of pre-cracks were examined, as shown in Figure 8. In each case the cracks were propagated by displacing the vertical boundaries of the cracked body outwards. In this manner simple tension was simulated, with no shear element. The propagation of the cracks is shown in Figure 9 and generally follows the pattern found experimentally by Fender et al. [39] and Cortet et al. [38].

\subsection{Exploring Settlement of Masonry}

Simulations of a simplified schematic of a masonry wall were carried out on 310 a geometry shown in Figure 10 The masonry units are indicated in red and the 


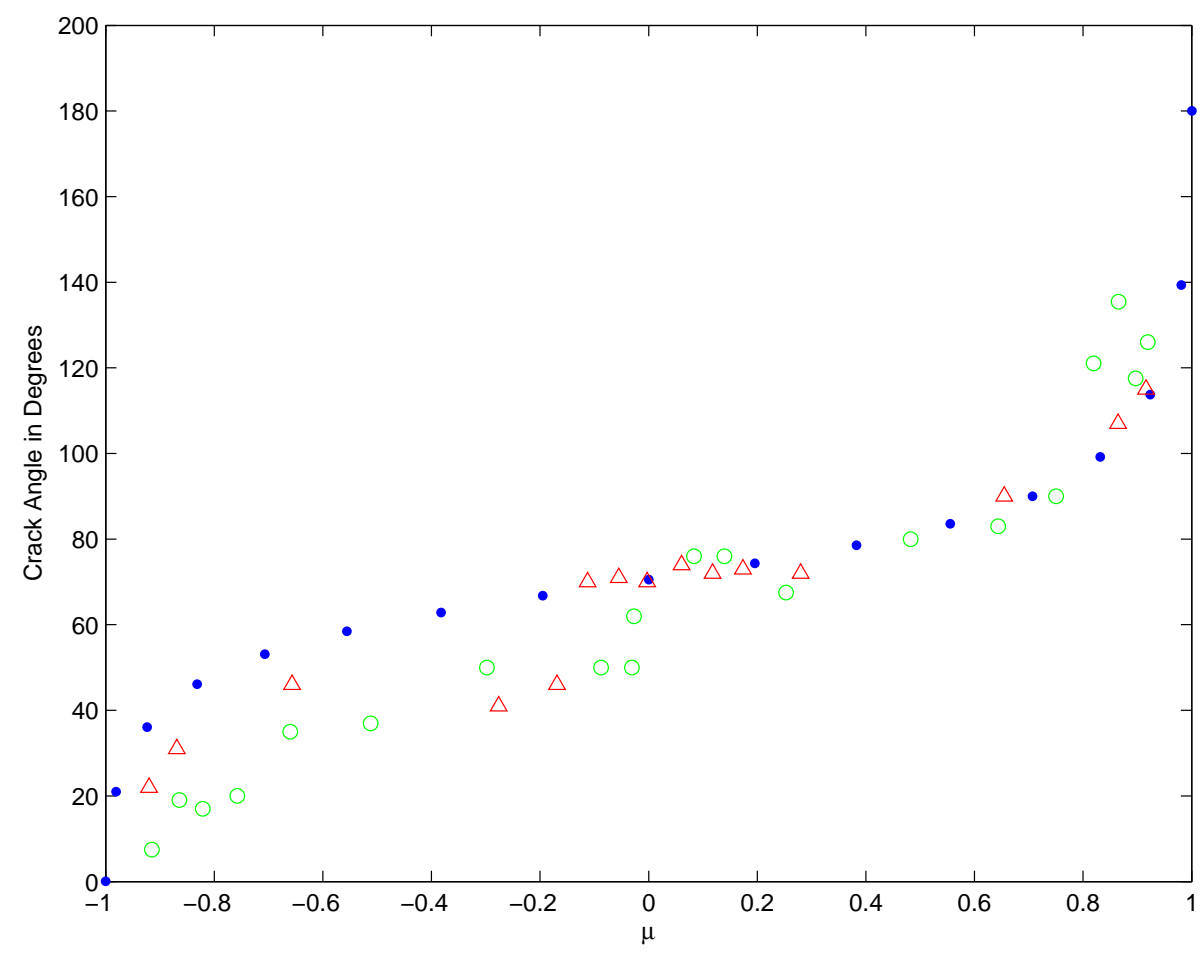

Figure 7: Analysis of kinked crack. $\mu=\frac{\left(\frac{K_{I}}{K_{I I}}\right)}{\sqrt{\left(\frac{K_{I}}{K_{I I}}\right)^{2}+1}}$. Blue dots show the theoretical predictions. Predictions using the model (for two different assemblies) are shown as green circles and red triangles.

central mortar joint between them is shown in green. The upper surface was displaced downwards to mimic a downward acting uniformly distributed load (UDL), and the support to the central portion was removed leaving supports only at the ends, as indicated by the blue dots. The strength of the elements in the mortar were set to different fractions of those in the masonry units. It should be noted that the supports are not symmetrical so the maximum bending moment does not coincide with the position of the mortar joint.

These show that, when the mortar strength is much lower than the that of the masonry, the crack is confined to the mortar joint; but as the strength of the 320 mortar increases, the crack first starts to diverge from its initiation point within the mortar into the masonry (see Figure 10(d)) and, as the strength increases again, the crack position moves to a point determined by the geometry and uninfluenced by the relative strengths. The tendency of the crack to curve as it extends into the masonry unit is noted and conforms to observations [36, 58].

325 The simulation shown in Figure 10(d) was allowed to run for a greater number of increments than the others to show the tension softening of the material as 


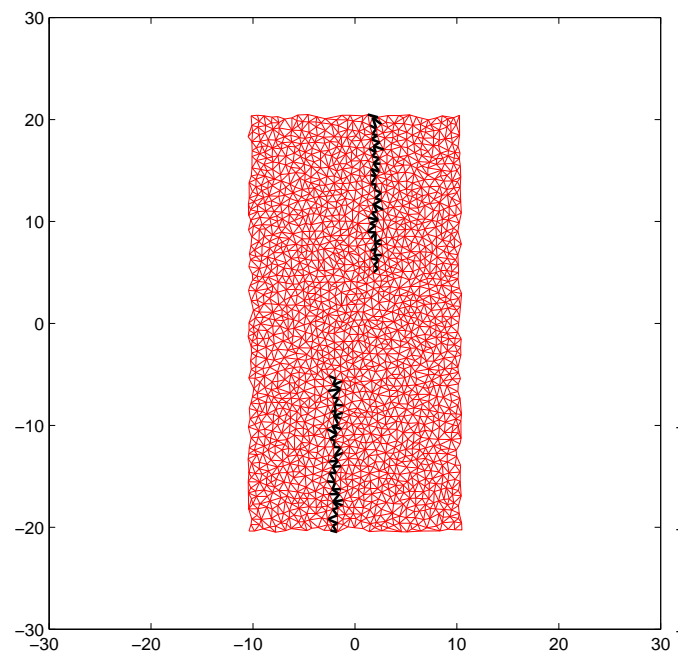

(a)

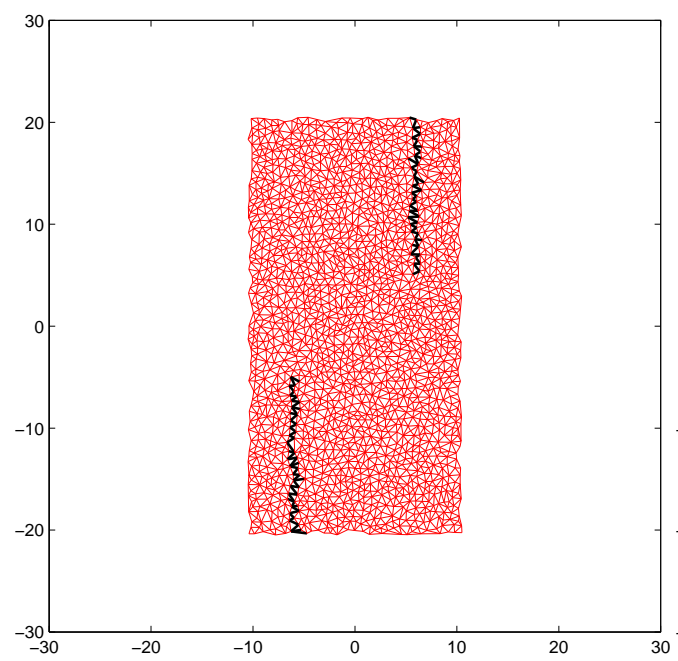

(c)

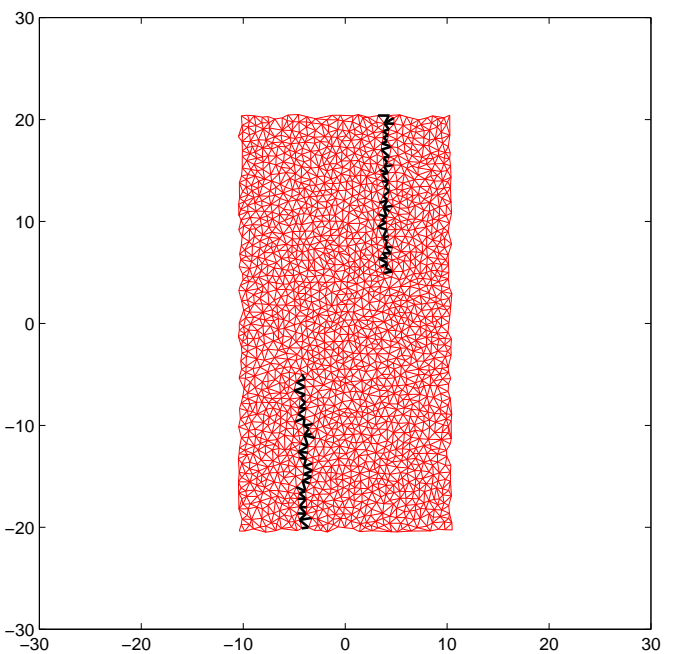

(b)

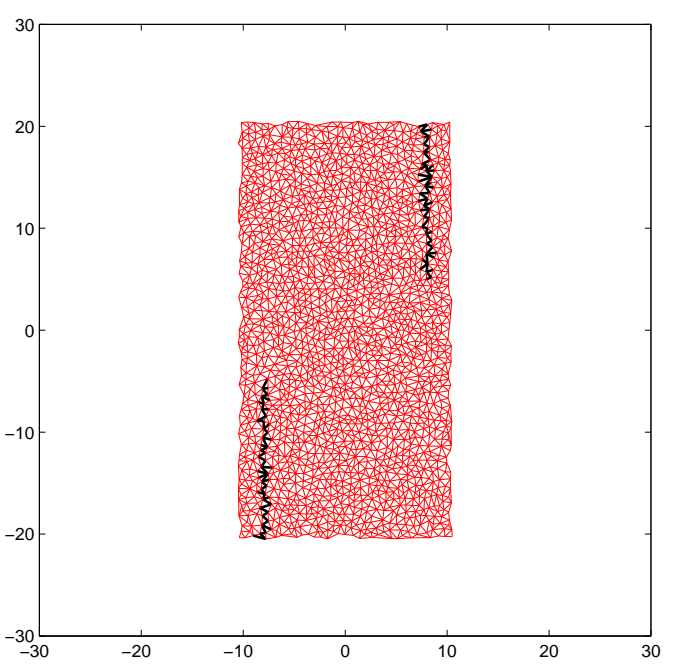

(d)

Figure 8: Arrangement of opposing off-set pre-cracks. Cracks shown as thick black lines. 


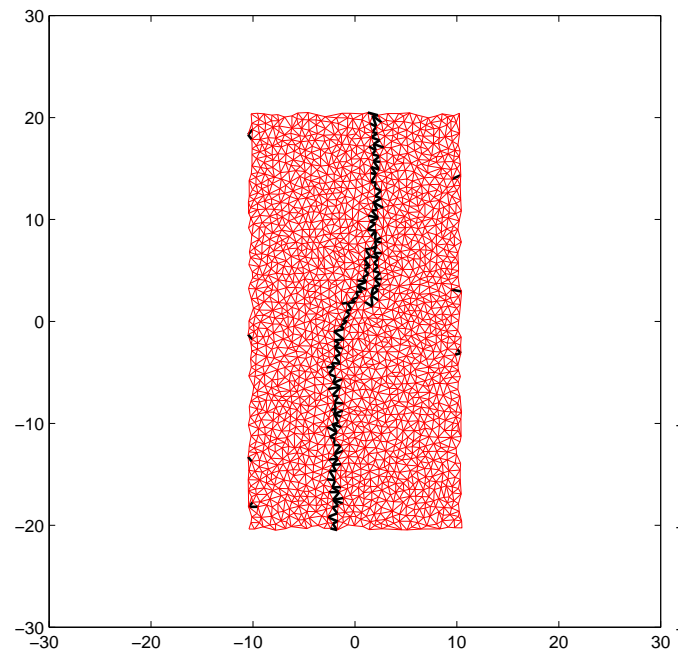

(a)

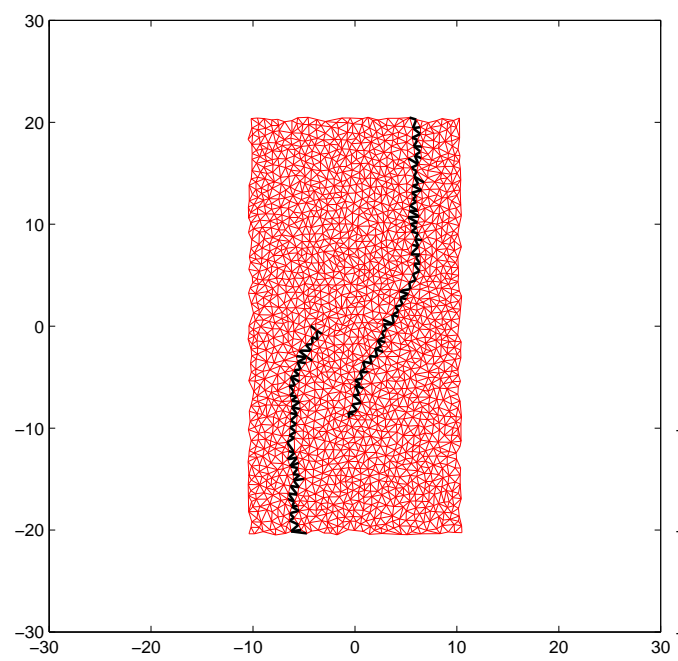

(c)

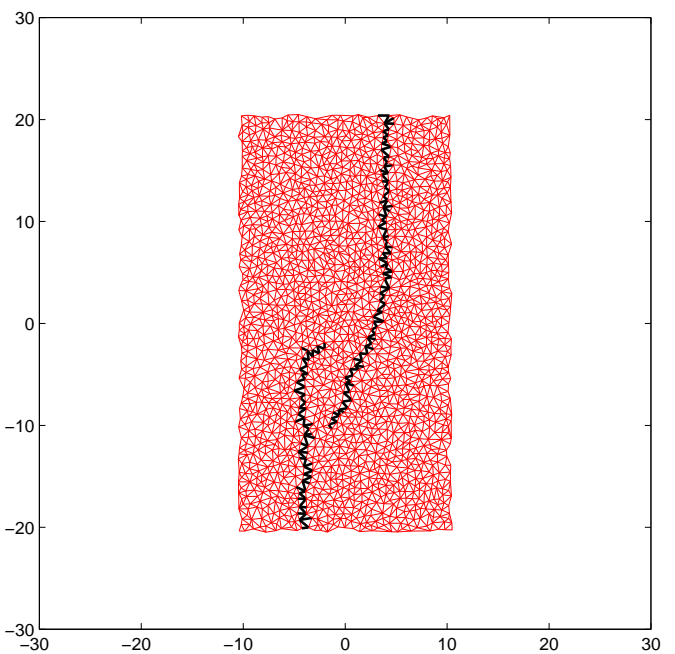

(b)

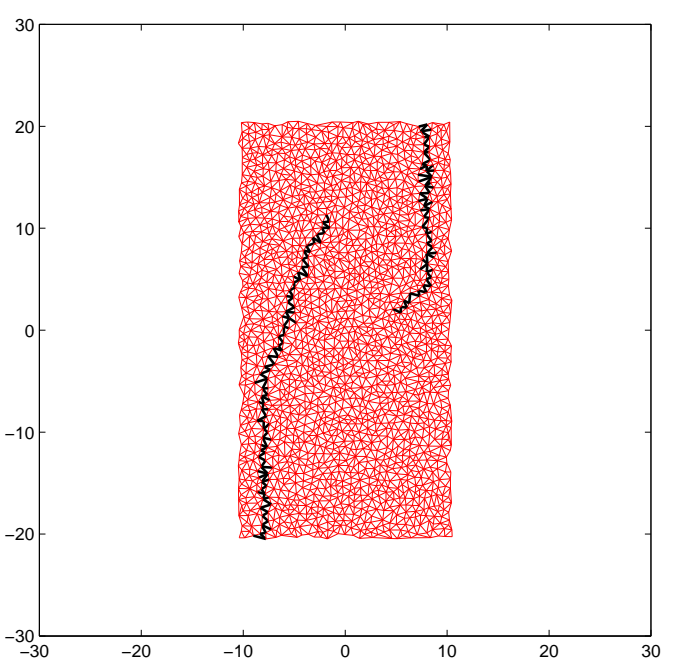

(d)

Figure 9: Propagation of Opposing Off-set Pre-Cracks shown in Figure 8 Cracks shown as thick black lines. 


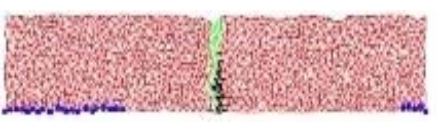

(a)

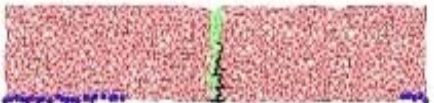

(c)

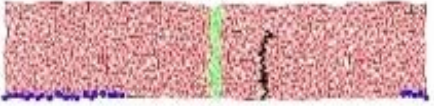

(e)

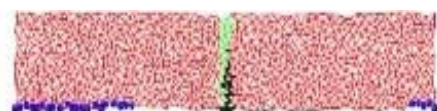

(b)

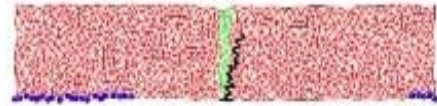

(d)

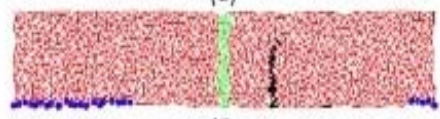

(f)

Figure 10: Idealised Schematic of Masonry (shown red) with central mortar (shown green) loaded by displacing the upper surface downwards to mimic a UDL on the upper surface and supported as indicated by blue dots. It should be noted that the supports are not symmetrical and so the maximum moment does not occur at the mid-point of the beam. Cracks shown as thick black lines. (a) Mortar strength at $10 \%$ of masonry strength; (b) mortar strength at $20 \%$ of masonry strength; (c) mortar strength at $40 \%$ of masonry strength; (d) mortar strength at $60 \%$ of masonry strength; (e) mortar strength at $80 \%$ of masonry strength; (f) mortar strength at $100 \%$ of masonry strength (f). 
is illustrated in Figure 11 .

\subsection{Exploring the Behaviour of a Flat Arch}

A simulation of a flat arch was carried out as illustrated in Figure12 Motion

330 upper surface near mid-span were displaced downwards to mimic a central patch load. The strength of the mortar was kept at $10 \%$ of the masonry strength and, as can be seen, the cracks occur in the tension zones and are mainly confined to the mortar joints. As the cracks develop they show the tendency to curve that

\subsection{Simulating Standard Tests}

A diametrical compression test (or cylinder splitting test) was simulated using the model and produced the classic crack pattern as shown in Figure 13(a). The load vs displacement curve produced by this simulation is shown in Figure 13(b). It should be recalled that the simulation does not relate to any specific material and so the numerical values are not significant and serve only to aid comparison. The curve clearly shows the strain softening, known to occur post-peak load in brittle materials of the type being considered.

A four point bend test was simulated as shown in Figure 14(a). The two nodes on the upper surface indicated by the blue circles were displaced vertically downwards while the two nodes on the lower surface indicated by the green circles were prevented from moving vertically. Two simulations were run, horizontally restraining each of the lower nodes in turn, but there was no noticeable difference in the results. The load vs displacement curve produced by this simulation is shown in Figure 14(b). As before this does not relate to any specific material. The plot clearly shows the expected strain softening.

\section{Concluding Remarks}

This paper presents a two dimensional lattice model that could easily be extended into three dimensions and that captures the essential behaviour of weakly bonded granular materials. In the context of this paper, 'weakly bonded' refers to the situation where fracture of the material would occur only by rupture of the inter-granule bonds, leaving the granules substantially intact. It has been demonstrated (see Figures 5 and 7 ) that the model obeys the fundamental laws of linear elastic fracture mechanics. Inter-granule bond stiffnesses that 360 more closely mimic the interaction between bonded granules than the more commonly assumed springs; bars or Euler-Bernoulli beams have been used to produce realistic simulations of the response of some brittle materials to load.

It is clear (see Figure 2) that the model can satisfactorily simulate an isotropic material and it is clear from Subsection 4.2 that anisotropy could be introduced. Simulations conducted using the model confirm the experimentally observed behaviour that, in bonded granular materials, crack branching and crack merging rarely happen as a consequence of statically applied load. 


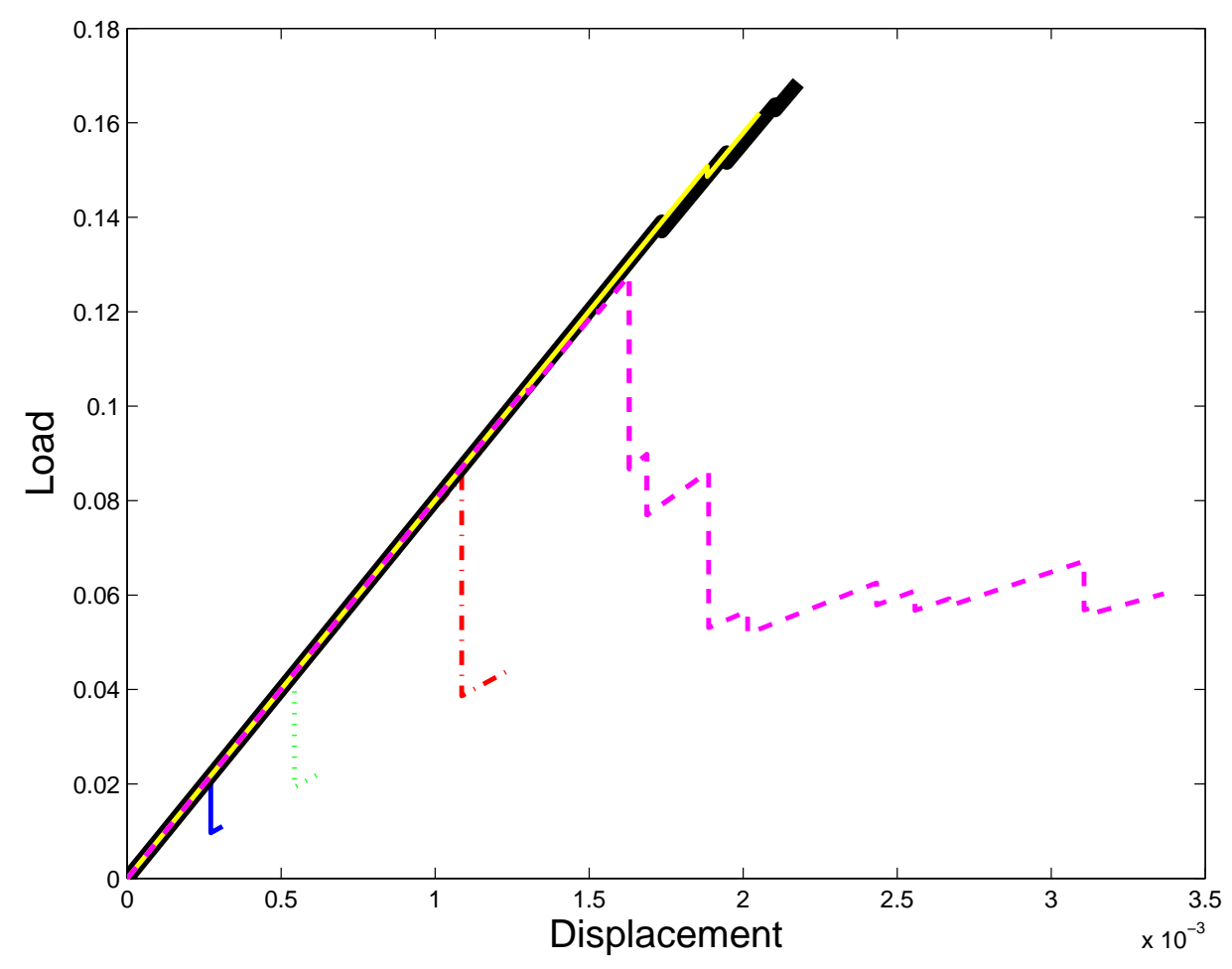

Figure 11: Load versus displacement curves for the simulations shown in Figure 10. The short, solid blue line relates to a mortar strength at $10 \%$ of the masonry strength; the doted green line relates to a mortar strength at $20 \%$ of the masonry strength; the red dash-dot line relates to a mortar strength at $40 \%$ of the masonry strength; the dashed magenta line relates to mortar strength at $60 \%$ of the masonry strength (this simulation was run for a greater number of increments than the others to show the full crack development); the solid thick black line relates to a mortar strength at $80 \%$ of the masonry strength; the thin yellow line relates to a mortar strength at $100 \%$ of the masonry strength. The simulations to which this figure relates were conducted for comparison purposes only and did not relate to any specific material. It follows that the numerical values shown for the load and the displacement are not significant. The grain size is, on average, about $1.2 \%$ of the length of the wall, and the area of the contacts bonding the grains is, on average, about $1.9 \%$ of the surface area of the each of the grains that it connects. 


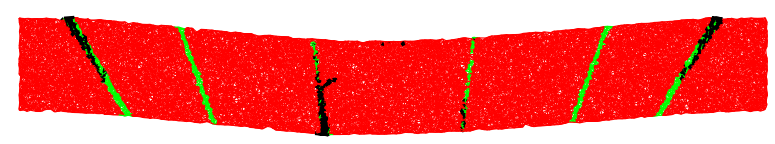

Figure 12: Idealised schematic of a flat arch restrained at the ends from moving outwards or downwards and loaded by vertically displacing two nodes on the upper surface to mimic a small patch of distributed load. The mortar (shown green) strength is $10 \%$ of the masonry (shown red) strength. Cracks shown as thick black lines.

The tendency of off-set opposing cracks to 'bypass' each other, which has been observed by others 39, 38], is confirmed by simulations presented here.

Simulations of an idealised portion of masonry with non-symmetric supports and subjected to load and settlement of supports illustrates the significance of the relative strengths of the mortar compared to the masonry units and the influence of that on the resulting crack pattern. These simulations indicate that the model presented here, although developed to investigate cracking confined 375 to the bonds between granules, could also be used to investigate through granule cracking. A clear understanding of the features of bonded granular materials that control cracking is very important for many engineering activities, including construction, tunnelling, drilling, hydraulic fracturing and mechanical forming. The tests and simulations presented here illustrate the utility of the model presented in this paper for investigating cracking in bonded granular assemblies.

[1] B. Chiaia, A. Vervuurt, J. G. M. van Mier, Lattice model evaluation of progressive failure in disordered particle composites, Engineering Fracture Mechanics 57 (2/3) (1997) 301-318.

[2] B. K. Raghuprasad, D. N. Bhat, G. S. Bhattacharya, Simulation of fracture in a quasi-brittle material in direct tension - a lattice model, Engineering Fracture Mechanics 61 (1998) 445-460. 


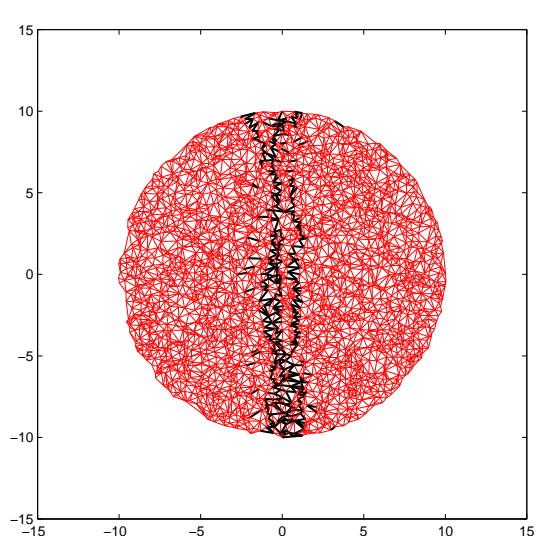

(a)

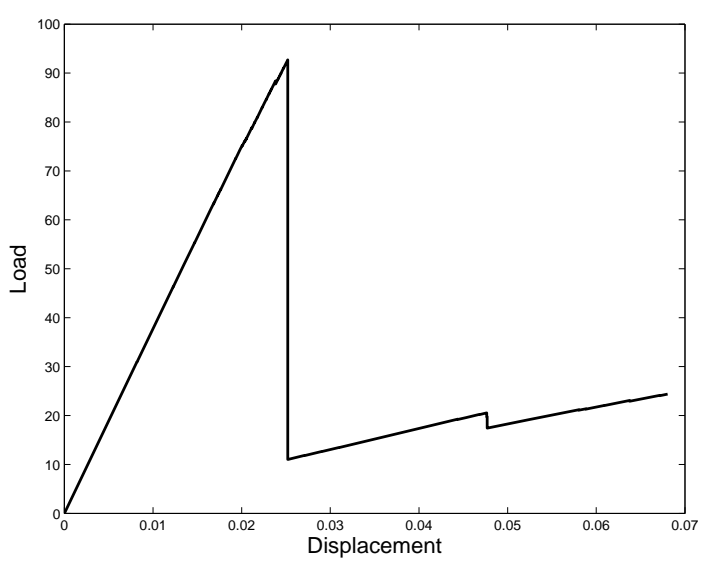

(b)

Figure 13: (Diametrical compression test. (a) Cracked cylinder after loading.(b) Load vs displacement curve. This simulation does not relate to any specific material and so the numerical values shown for the load and the displacement are not significant. The grain size is, on average, about $1.8 \%$ of the diameter of the cylinder, and the area of the contacts bonding the grains is, on average, about $1.85 \%$ of the surface area of the each of the grains that it connects.

[3] V. Topin, J. Y. Delenne, F. Radja, L. Brendel, F. Mabille, Strength and failure of cemented granular matter, The European Physical Journal E 23 (2007) 413-429.

[4] N. P. Kruyt, L. Rothenburg, Micromechanical bounds for the effective elastic moduli of granular materials, International Journal of Solids and Structures 39 (2002) 311-324.

[5] G.-F. Zhao, A. R. Russell, X. Zhao, N. Khalili, Strain rate dependency of uniaxial tensile strength in gosford sandstone by the distinct lattice spring model with x-ray micro ct, International Journal of Solids and Structures 51 (2014) 1587-1600.

[6] R. Katsman, E. Aharonov, H. Scher, Numerical simulation of compaction bands in high-porosity sedimentary rock, Mechanics of Materials 37 (2005) $143-162$.

[7] R. Katsman, E. Aharonov, A study of compaction bands originating from cracks, notches, and compacted defects, Journal of Structural Geology 28 (2006) 508-518.

[8] D. O. Potyondy, P. A. Cundall, A bonded-particle model for rock., International Journal of Rock Mechanics and Mining Sciences 41 (2004) 1329-1364. 


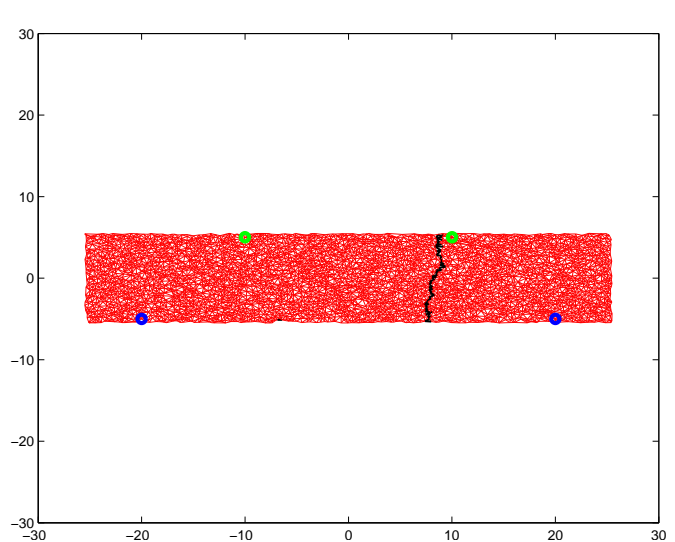

(a)

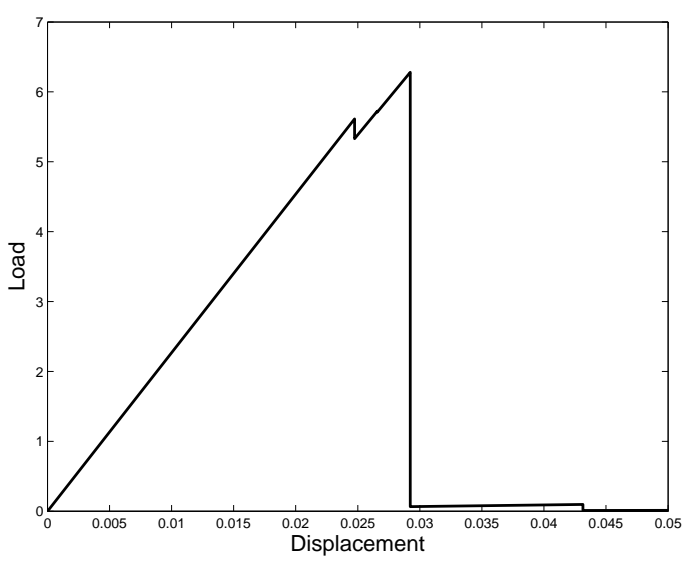

(b)

Figure 14: Four point bend test. Downwards point loads applied (by means of fixed displacements) on the upper surface at the positions indicated by the blue circles. Vertical displacement was prevented at the positions on the lower surface indicated by the two green circles and horizontal displacement was prevented at the green circle on the right hand side. (a) Cracked beam after loading. (b) Load vs displacement curve. This simulation does not relate to any specific material and so the numerical values shown for the load and the displacement are not significant. The grain size is, on average, about $0.8 \%$ of the length of the beam, and the area of the contacts bonding the grains is, on average, about $0.06 \%$ of the surface area of the each of the grains that it connects. 
[20] B. L. Karihaloo, A. Carpinteri, M. Elices, Fracture mechanics of cement mortar and plain concrete, Advanced Cement Based Materials 1 (1993) 92-105.

[21] L. Pchenik, H. Levine, D. Kessler, Steady-state mode i cracking in a viscoelastic triangular lattice, Journal of the Mechanics and Physics of Solids 50 (2002) 583-613. 
[22] S. I. Heizler, D. A. Kessler, H. Levine, Propagating mode-i fracture in amorphous materials using the continuous random network model, Physical Review E 82 Issue 2 (Article 026102 Part 2).

[23] K. Kim, J. E. Bolander, Y. M. Lim, Failure simulation of rc structures under highly dynamic conditions using random lattice models, Computers and Structures 125 (2013) 127-136.

[24] M. Yip, Z. Li, B. S. Liao, J. E. Bolander, Irregular lattice models of fracture of multiphase particulate materials, International Journal of Fracture 140 (2006) 113-124.

[25] N. Cho, C. D. Martin, D. C. Sego, A clumped particle model for rock, International Journal of Rock Mechanics and Mining Sciences 44 (2007) 997-1010.

[26] K. Mair, S. Abe, Breaking up: Comminution mechanisms in sheared simulated fault gouge, Pure and Applied Geophysics 168 (2011) 2277-2288.

[27] J. Elias, H. Stang, Lattice modeling of aggregate interlocking in concrete, International Journal of Fracture 175 (2012) 1-11, doi 10.1007/s10704-0129677-3.

[28] C. Thornton, D. J. Barnes, Computer simulated deformation of compact granular assemblies, Acta Mechanica 64 (1) (1986) 45-61.

[29] H. Ouadfel, L. Rothenburg, Stress-force fabric relationship for assemblies of ellipsoids, Mechnics of Materials 33 (2001) 201-221.

[30] N. P. Kruyt, I. Agnolin, S. Luding, L. Rothenburg, Micromechanical study of elastic moduli of loose granular materials, Journal of the Mechanics and Physics of Solids 58 (2010) 1286-1301.

[31] D. Andre, M. Jebahi, J.-L. Charle, J. Néauport, Using the discrete element method to simulate brittle fracture in the indentation of a silica glass with a blunt indenter, Computation methods in applied mechanics and engineering 263 (2013) 136-147.

[32] C. M. Sands, R. J. Henderson, H. W. Chandler, A three dimensional computational model of the mechanical response of a dual-phase ceramic, Computational Materials Science 39 (2007) 862-870.

[33] H. Y. Liu, M. Roquette, S. Q. Kou, P.-A. Lindqvist, Characterization of rock heterogeneity and numerical verification, Engineering Geology 72 (2004) 89-119.

[34] L. Li, Q. Meng, S. Wang, A numerical investigation of the hydraulic fracturing behaviour of conglomerate in glutenite formation, Acta Geomechanica 8 (2013) 597-618. 
[35] S. Y. Wang, S. W. Sloan, H. Y. Liu, C. A. Tang, Numerical simulation of the rock fragmentation process induced by two drill bits subjected to static and dynamic (impact) loading., Rock Mechanics and Engineering 44 (2011) 317-332.

[36] F. Erdogan, G. C. Sih, On the crack extension in plates under plain loading and transverse shear, Transactions of the American Society of Mechanical Engineers, Journal of Basic Engineering 85 (1963) 519-527.

[37] S. Melin, Why do cracks avoid each other?, International Journal of Fracture 23 (1) (1983) 37-45.

[38] P.-P. Cortet, G. Huillard, L. Vanel, S. Ciliberto, Attractive and repulsive cracks in a heterogeneous material, Journal of Statistical Mechanics: Theory and Experiment Article P10022.

[39] M. L. Fender, F. Lechenault, D. K. E., Universal shapes formed by two interacting cracks, Physical Review Letters 105 Issue 12, article 125505.

[40] G. N. Pande, J. X. Liang, J. Middleton, Equivalent elastic moduli for brick masonry, Computers and Geotechnics 8 (1989) 243-265.

[41] J. S. Lee, G. N. Pande, J. Middleton, B. Kralj, Numerical modelling of brick masonry panels., Computers and Structures.

[42] G. Alpa, I. Monetto, Microstructural model for dry block masonry walls with in-plane loading., Journal of the Mechanics and Physics of Solids 42 (7) (1994) 1159-1175.

[43] A. Anthoine, Derivation of the in-plane elastic characteristincs of masonry through homogenization theory., International Journal of Solids and Structures 32 (2) (1995) 137-163.

[44] R. Luciano, E. Sacco, Homogenization technique and damage model for old masonry material., International Journal of Solids and Structures. 34 (24) (1997) 3191-3208.

[45] K. Sab, Yield design of thin periodic plates by a homogenization technique and an application to masonry walls., C. R. Mecanique 331 (2003) 642-646.

[46] A. Cecchi, K. Sab, A comparison between a 3d discrete model and two homogenised plate models for periodic elastic brickwork., International Journal of Solids and Structures 41 (2004) 2259-2276.

[47] A. Cecchi, K. Sab, A homogenized lovekirchhoff model for out-of-plane loaded random 2d lattices: Application to quasi-periodic" brickwork panels, International Journal of Solids and Structures 46 (2009) 2907-2919.

[48] G. Milani, Simple lower bound limit analysis homogenization model for in-and-out-of-plane loaded masonry walls, Construction and Building Materials 25 (2011) 4426-4443. 
[49] M. Hammoud, K. Sab, D. Duhamel, A coupled discrete/continuous method for computing lattices. application to masonry-like structure, International Journal of Solids and Structures.

520 [50] S. Casolo, G. Milani, A simplified homogenization-discrete element model for the non-linear static analysis of masonry walls out-of-plane loaded, Engineering Structures 32 (number) (2010) 2352-2366.

[51] J. R. Barber, Elasticity (Solid Mechanics and its Application), Springer, 1992.

${ }_{525}$ [52] K. L. Johnson, Contact Mechanics, paperback edition Edition, Cambridge University Press, Cambridge, 1987, section 7.2.

[53] R. K. Livesley, Matrix Methods of Structural Analysis, Pergamon Press, Oxford, 1975.

[54] M. H. Adaiem, Wellbore integrity in shale strata, Ph.D. thesis, University of Aberdeen (2010).

[55] M. L. Williams, On the stress distribution at the base of a stationary crack, Journal of Applied Mechanics 24 (1957) 109-114.

[56] J. R. Rice, Discussion on stresses in an infinite strip containing a semiinfinite crack, Transactionso of the American Society of Mechanical Engineers (1967) 248-249.

[57] W. G. Knauss, Stresses in an infinite strip containing a semi-infinite crack, ASME Journal of Applied Mechanics 33 (1966) 356-362.

[58] B. Cotterell, J. R. Rice, Slightly curved or kinked cracks, International Journal of Fracture 16 (2) (1980) 155-169. 